Revue des patrimoines

\title{
Le cheval et les ressources numériques de Gallica, patrimoine diffus d'une bibliothèque coopérative
}

\section{Arnaud Dhermy}

\section{(2) OpenEdition}

\section{Journals}

Édition électronique

URL : http://journals.openedition.org/insitu/9740

DOI : $10.4000 /$ insitu. 9740

ISSN : 1630-7305

\section{Éditeur}

Ministère de la culture

Référence électronique

Arnaud Dhermy, "Le cheval et les ressources numériques de Gallica, patrimoine diffus d'une bibliothèque coopérative », In Situ [En ligne], 18 | 2012, mis en ligne le 31 juillet 2012, consulté le 21 avril 2019. URL : http://journals.openedition.org/insitu/9740 ; DOI : 10.4000/insitu.9740

Ce document a été généré automatiquement le 21 avril 2019

\section{(c) $($ i) $(9)$}

In Situ Revues des patrimoines est mis à disposition selon les termes de la licence Creative Commons Attribution - Pas d'Utilisation Commerciale - Pas de Modification 4.0 International. 


\title{
Le cheval et les ressources numériques de Gallica, patrimoine diffus d'une bibliothèque coopérative
}

\author{
Arnaud Dhermy
}

1 Amorcée à partir de 1991 et présente sur Internet à partir de 1997, Gallica, la bibliothèque numérique de la Bibliothèque nationale de France, est un fonds d'ouvrages à la fois d'intérêt général, spécifiques, ou encore difficiles d'accès. Son développement se réalise à partir de plusieurs marchés distincts de numérisation: livres et revues; presse quotidienne d'intérêt national; documents précieux: manuscrits, cartes et plans, estampes, photographies, affiches, partitions, documents sonores. Certains critères, comme la rareté ou la spécificité thématique et disciplinaire, amènent par ailleurs des rapprochements avec d'autres institutions, ce qui fait de Gallica un projet collectif qui connaît une extension collaborative; en cela elle illustre le décret constitutif de la BnF sur les points de la « coopération avec les autres bibliothèques et centres de recherche et de documentation français», et de la «mise en commun des ressources documentaires des bibliothèques françaises $"$.

2 En dépit de cette démarche d'accroissement tant encyclopédique que volumétrique, Gallica ne possède pas de collections importantes ni cohérentes sur la thématique du cheval. Cela tient à un éclatement des collections abordant le patrimoine équestre dans les différentes rubriques de la cotation de la BnF, qui se traduit par une dispersion physique rendant difficile l'exploitation des collections imprimées dans le cadre d'un programme homogène. Cet état de fait tient aussi à l'absence, jusqu'à présent, de partenariats documentaires et scientifiques dans ce domaine.

3 Le patrimoine équestre représente cependant dans Gallica un corpus d'environ 220 titres, que l'on pourrait répartir en sept rubriques : l'équitation ; l'élevage ; le cheval de guerre ; l'hippiatrie; le cheval comme moyen de transport; le jeu et les fêtes; les institutions constituées autour du cheval. Les ouvrages en ligne peuvent aussi bien être des éditions 
originales de grands traités (Pluvinel, La Broue), que des rééditions (La Guérinière), ou des études et manuels du XIX siècle. Plusieurs catalogues de carrossier (Kellner), des albums de photographies hippiques (Delton) édités dans la seconde moitié du XIX ${ }^{\mathrm{e}}$ siècle viennent enrichir ces références sur le plan graphique.

4 À ces ressources imprimées s'ajoutait en 2011 un ensemble de plus de 250 estampes et clichés provenant des collections spécialisées de la $\mathrm{BnF}$ : statuaire; portraits équestres (Dürer, Delaroche, Odilon Redon...) ; scènes de fêtes ; études de maîtres ; mais aussi des esquisses (les chevaux de Marly), des relevés de frontons et bas-reliefs, des planches anatomiques, des cours de dessin, des copies d'enluminures, des scènes de fête tirées des collections d'estampes historiques de Gaignières, Destailleur ou De Vinck, etc., et enfin des illustrations d'éditions modernes, comme les recueils de fables d'Ésope ou La Fontaine par Oudry.

5 Au-delà des ouvrages explicitement consacrés au patrimoine équestre, Gallica de par son volume et sa diversité témoigne cependant d'une société française dans laquelle le cheval constitue un élément essentiel et structurant. Celui-ci est présent dans la vie économique (les revues d'agriculture, les essais sur le commerce...), à l'armée (les textes de réglementation militaire), sur les routes (les dictionnaires des postes, les traités de louage...), dans la législation et la vie publique (les publications officielles, notamment celles des conseils généraux,...), dans les jeux et dans les spectacles (les revues sportives), comme dans l'archéologie (les publications des sociétés savantes régionales) et enfin dans l'art (voir l'étude sur « Le canon hippique » publiée dans la Gazette des beaux arts).

6 Le patrimoine équestre est donc bien présent dans Gallica, mais par le biais d'une dissémination documentaire plutôt que dans un corpus clairement identifié : ce sont aussi plus de 7200 références figurant dans des tables des matières et dans le légendage des images ; et surtout plus de 80000 occurrences dans les contenus des textes eux-mêmes.

7 Cet ensemble ne saurait cependant représenter un seuil critique satisfaisant, c'est pourquoi la BnF souhaite ouvrir pour les livres et les revues son marché de numérisation afin d'améliorer sensiblement l'offre existante, au-delà du seuil de l'anthologie. Cette démarche de la BnF peut aussi se concrétiser par un soutien à la numérisation d'exemplaires plus caractéristiques ou mieux conservés que ceux dont elle dispose, comme cette édition du Cours d'hippiatrique de Lafosse, présentée le 15 juin 2011 au château de Pompadour au cours de l'exposition «Le parfait maréchal : une histoire des maréchaux-ferrants » organisée par Joëlle Cartigny, conseiller Livre et Lecture à la DRAC du Limousin, édition issue des collections de la Médiathèque de Guéret et qui bientôt enrichira Gallica.

8 Cette démarche documentaire peut enfin se réaliser par un rapprochement entre réservoirs numériques déjà constitués. Elle permet à des collections en ligne, comme celles de l'École vétérinaire d'Alfort, d'être plus particulièrement interrogeables depuis Gallica sur tel ou tel sujet, grâce à une interopérabilité entre les deux bibliothèques à partir des seules notices des ouvrages (ce «moissonnage» est réalisé à partir du protocole OAI-PMH). Cet échange permet en retour aux autres bibliothèques numériques de bénéficier des collections présentes sur Gallica, qui peuvent être sélectionnées et signalées en fonction de leurs propres thématiques d'expertise (voir le portail documentaire du Muséum national d'histoire naturelle, les portails régionaux Aquitaine Patrimoines, Manioc, etc.). 
Gallica est en effet une plate-forme donnant accès non seulement aux collections de la BnF mais aussi à des sélections proposées par ses partenaires: en 2011 plus de 63000 documents provenant par interopérabilité d'autres bibliothèques sont visibles dans Gallica, mais aussi 7500 autres documents extérieurs qui ont été directement numérisés par la $\mathrm{BnF}$ et mis en ligne dans sa bibliothèque numérique. Ce dernier volume est particulièrement appelé à s'accroître avec l'ouverture du marché de numérisation des livres et revues de la BnF aux collections de ses partenaires pour les années allant de 2011 à 2014.

10 La dimension collective de Gallica est une réalité - vitrine de la francophonie sur Internet, bientôt visible sur Europeana - mais ses thématiques restent encore largement à développer, à diversifier. La coopération numérique sur les livres et les revues est une démarche que la BnF propose à l'ensemble des structures disposant de fonds patrimoniaux d'intérêt national, et libres de droits, quels que soient le statut de ces structures, leur localisation et l'ampleur de leurs collections, quel que soit leur niveau d'engagement et les modalités de leur collaboration (scientifique, technique ou stratégique). L'objectif est de tendre vers une bibliothèque numérique appartenant à tous et à chacun, que chaque partenaire puisse valoriser en fonction de ses objectifs, de son public ou de son territoire.

11 Cette démarche est à bâtir collectivement depuis la sélection des corpus et la numérisation, jusqu'à l'éditorialisation, la diffusion et la valorisation, avec une recherche d'efficacité financière et documentaire. Elle ambitionne de produire dans le domaine retenu un ensemble de livres et de revues des plus vastes, en organisant les apports de chaque partenaire autour du noyau constitué par la BnF dans le cadre de la numérisation de ses collections.

12 Les ressources ainsi rassemblées dans Gallica deviennent un réservoir disponible pour un portail global susceptible de fédérer ou d'articuler tous les supports et toutes les ressources en ligne. C'est bien cet objectif que la BnF se propose d'atteindre, en faveur du patrimoine équestre et des institutions documentaires qui le représentent.

13 Ce projet autour du patrimoine équestre pourrait se décliner en différents partenariats numériques, centrés à la fois autour d'expertises thématiques et de ressources documentaires disponibles. L'hippologie et l'hippiatrie pourront être abordées avec l'École nationale vétérinaire d'Alfort, déjà partenaire de la BnF pour la numérisation. À moyen terme pourra aussi voir le jour un projet autour de l'équitation avec l'École nationale d'Équitation et la Ville de Saumur; un autre encore autour de la maréchalerie et plus largement de l'équipement et ses métiers avec le pôle associé régional de la BnF en Limousin ; enfin autour de la cavalerie avec les établissements documentaires dépendant du ministère de la Défense, également partenaire de la BnF. D'autres partenariats pourraient aussi être suscités, notamment autour de l'élevage, des transports et du matériel hippomobile, de l'hippisme et du turf, des fêtes et de l'apparat... Ces projets auront à se coordonner dans un contexte plus global autour du patrimoine équestre. Une base de données créée par l'École nationale d'Équitation, dans le cadre du portail documentaire qu'elle anime, et développée en concertation avec Gallica, rassemblera les différentes propositions et réalisations autour de cette thématique. Ce dispositif permettra d'éviter les doublonnements mais surtout facilitera les compléments et le comblement d'éventuelles lacunes d'un projet à l'autre. 
14 C'est donc, pour ce projet soutenu par le marché de numérisation de la BnF, une mutualisation des collections à laquelle on doit aspirer, de manière à constituer un autre "patrimoine immatériel» aussi cohérent que possible. Par delà les thématiques et expertises évoquées, il aura en particulier à articuler sa valorisation autour d'outils documentaires structurants. En premier lieu, il s'agira de rassembler les grandes revues du domaine, lacunaires en bien des lieux et difficiles à dépouiller, qu'elles soient techniques, professionnelles mais aussi culturelles (La France chevaline, le Journal des haras ...). De même, les publications officielles en rapport avec le monde du cheval : almanachs, annuaires hippiques, calendriers des courses, statistiques, registres imprimés de généalogies et stud-books, congrès hippiques... Enfin un ensemble aussi important que possible de traités et manuels (élevage, conduite...) qu'il faudra enrichir par les approches locales et régionales.

\section{RÉSUMÉS}

Riches de plus d'un million et demi de documents, les collections de Gallica, la bibliothèque numérique de la Bibliothèque nationale de France, sont fortement imprégnées par le cheval même si elles ne constituent pas pour autant un important centre de ressources en la matière. La BnF cependant souhaite accroître l'offre actuelle au-delà du stade de l'anthologie. À travers Gallica, bibliothèque numérique coopérative, elle vise à bâtir collectivement un autre patrimoine immatériel qui tienne compte à la fois des expertises et des collections de chacun de ses partenaires.

Rich in more than 1,5 million documents, the collections of Gallica, the digital library of the National Library of France (NLF), denote a strong impregnation of the horse even if they do not constitute an important center of resources on the subject. The NLF however wants to increase the current offer beyond the stage of the anthology. Through Gallica, a cooperative digital library, she aims at building collectively another intangible cultural heritage which takes into account, at the same time, expertises and collections of each of his partners.

\section{INDEX}

Mots-clés : numérisation, Gallica, mutualisation documentaire, programme de mise en ligne

\section{AUTEUR}

\section{ARNAUD DHERMY}

Bibliothèque nationale de France, Coordinateur scientifique au département de la Coopération, Service Gallica arnaud.dhermy@bnf.fr 